\title{
EFFECT OF LATTICE AND ELECTRON DISTRIBUTION IN ELECTRON-CLOUD INSTABILITY SIMULATIONS FOR THE CERN SPS AND LHC
}

\author{
E. Benedetto*, Politecnico di Torino, Italy, and CERN, Switzerland \\ G. Arduini, F. Roncarolo, F. Zimmermann, CERN, Switzerland \\ G. Rumolo, CELLS, Spain, G. Franchetti, GSI, Germany, K. Ohmi, KEK, Japan \\ T. Katsoueleas, A. Ghalam, B. Feng, USC, California, USA
}

\begin{abstract}
Several simulation codes have been adapted so as to model the single-bunch electron-cloud instability including a realistic variation of the optical functions with longitudinal position. In addition, the electron cloud is typically not uniformly distributed around the ring, as frequently assumed, but it is mainly concentrated in certain regions with specific features, e.g., regions which give rise to strong multipacting or suffer from large synchrotron radiation flux. Particularly, electrons in a dipole magnet are forced to follow the vertical field lines and, depending on the bunch intensity, they may populate two vertical stripes, symmetrically located on either side of the beam. In this paper, we present simulation results for the CERN SPS and LHC, which can be compared with measurements or analytical predictions.
\end{abstract}

\section{INTRODUCTION}

Many past studies of electron-cloud instabilities were performed considering a uniform electron distribution without magnetic field and a constant focusing lattice.

However, about $80 \%$ of the CERN SPS circumference is filled with bending magnets where the electron multipacting is higher than in field free regions. Therefore the behaviour of the electron cloud in the dipoles determines the characteristics of the induced instabilities and the same is expected for the LHC at injection. Figure 1 shows the electron flux in an SPS strip detector [1], installed inside a bending magnet, measured during machine studies in August ' 04 with LHC-type beam at $26 \mathrm{GeV}$. The electrons are mainly populating two vertical stripes. In the following section we study how the electron cloud characteristics affect the development of the instability using the code HEADTAIL, developed at CERN [2] for the study of transverse single bunch instabilities and emittance growth.

A second complication arises from the fact that the beam itself evolves in the lattice structure and, in particular, its transverse size varies according to the local beta function. As a result, the "pinch" of the electrons can be different at different locations of the machine. A first rough attempt to look at the effect of varying beam sizes was implemented in HEADTAIL, by allowing for different of beta-function values at the various "interaction points" between the cloud and the bunch, instead of considering only one average value. Studies and potential problems with this approach are discussed in the following.

A second step towards a more realistic lattice description has been to model the real evolution of the beam particles in a FODO cell. In a new version of HEADTAIL it is possible to follow the motion of the protons through the focusing and defocusing elements, while experiencing a quasicontinuous interaction with the electron cloud in between. In addition, it is now possible to choose the electron distribution independently in each element, for example as two vertical stripes in the dipoles. A great limitation is the excessive computing time needed when considering a large number of beam-electron interactions per turn.

For this reason, simulations with HEADTAIL can presently only be performed using a weak-strong model for the interaction between the cloud and the bunch [3]. This restriction is not too serious, since our main concern for the LHC and the SPS is the long-term emittance growth below the threshold of the fast TCMI like instability $[4,5]$, where weak-strong and strong-strong simulations yield nearly identical results [3]. Regardless, we are also pursuing an implementation of the same features in QuickPIC [6], which is a parallel plasma code and which will as well allow the strong-strong study of the coherent instability for a realistic lattice.

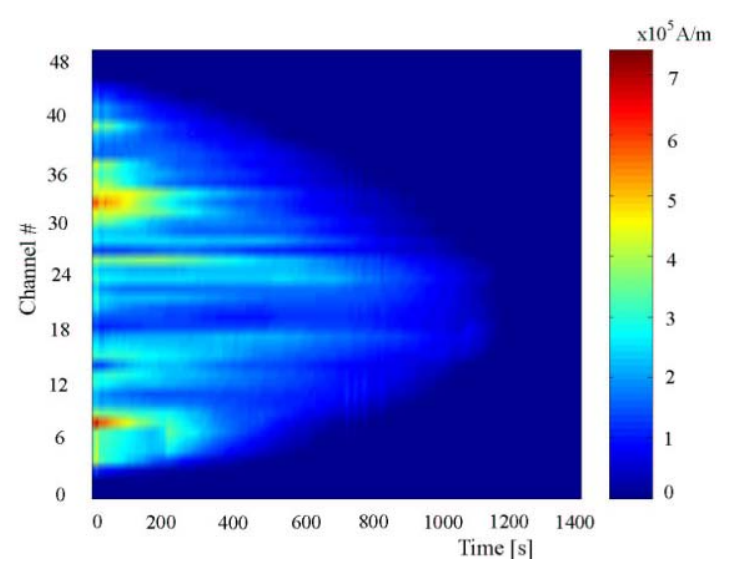

Figure 1: Electron cloud flux measurement using a strip detector in a dipole region of SPS, with LHC-type beam at $26 \mathrm{GeV}$

\footnotetext{
*elena.benedetto@cern.ch
} 
Table 1: Simulation parameters for LHC and SPS.

\begin{tabular}{lcc}
\hline & SPS & LHC \\
\hline bunch population, $N_{b}$ & $1.1 \times 10^{11}$ & $1.1 \times 10^{11}$ \\
av. beta function, $\beta_{x, y}[\mathrm{~m}]$ & 40 & 100 \\
rms bunch length, $\sigma_{z}[\mathrm{~m}]$ & 0.24 & 0.115 \\
rms beam size, $\sigma_{x, y}[\mathrm{~mm}]$ & 0.0021 & 0.884 \\
rms momentum spread, $\delta$ & 0.002 & $4.68 \times 10^{-4}$ \\
synchrotron tune, $Q_{s}$ & 0.0059 & 0.0059 \\
momentum compaction & $1.92 \times 10^{-3}$ & $3.47 \times 10^{-4}$ \\
circumference, $C[\mathrm{~m}]$ & 6911 & 26659 \\
nominal tunes, $Q_{x, y}$ & $26.185,26.13$ & $64.28,59.31$ \\
chromaticity, $Q_{x, y}^{\prime}$ & 2,2 & 2,2 \\
dispersion, $D$ [m] & 2.28 & 0 \\
relativistic factor, $\gamma$ & 27.728 & 479.6 \\
cavity voltage, $V$ [MV] & 2 & 8 \\
harmonic number, $h$ & 4620 & 35640 \\
\hline \# of macro-electrons & $10^{5}$ & $10^{5}$ \\
\# of macro-protons & $3 \times 10^{5}$ & $3 \times 10^{5}$ \\
\# of slices & 70 & 70 \\
\# of grid points & $128 \times 128$ & $128 \times 128$ \\
size of the grid & $10 \sigma_{x, y}$ & $10 \sigma_{x, y}$ \\
extent of the bunch in $z$ & $\pm 2 \sigma_{z}$ & $\pm 2 \sigma_{z}$ \\
\hline & & \\
\hline
\end{tabular}

\section{ELECTRON CLOUD IN A DIPOLE}

The parameters used for the simulations with the code HEADTAIL are listed in Table 1. Assuming that the electron cloud is concentrated in the bending regions of the SPS, we initialize the electrons as distributed in the form of two vertical stripes, with a Gaussian profile in $x$. The rms size of a stripe is taken to be $\sigma_{s}=6.85 \sigma_{x}$ and its distance from the origin $l=3.45 \sigma_{x}$, as inferred from the measurements (Fig. 1). The electrons are free to move along the vertical field lines, but their motion in the horizontal plane is frozen (strong magnetic field approximation). Figure 2 compares the development of the instability for a cloud in a dipole field and a field-free region. In the latter case, the initial electron distribution is uniform. The different curves refer to different values of the average electron density. At the same average density, the effect of the cloud is weaker in the dipole field. In particular, for $\rho_{e}=6 \times 10^{11} \mathrm{~m}^{-3}$, we notice a small growth over $40 \mathrm{~ms}$, while without magnetic field a fast TMCI-like instability develops. This difference can partly be explained by the presence of the stripes, which depletes the electron density at the center of the pipe. In addition, the electrons, following the field lines of the dipole, pinch towards the beam only in the vertical plane. Therefore, the accumulation of electrons inside the beam during the bunch passage is reduced as well. In the horizontal plane, as expected, no fast instability occurs, since the motion of the electrons is frozen in this direction. Neverthless, for high electron densities a significant emittance growth may probably be found even in this plane.
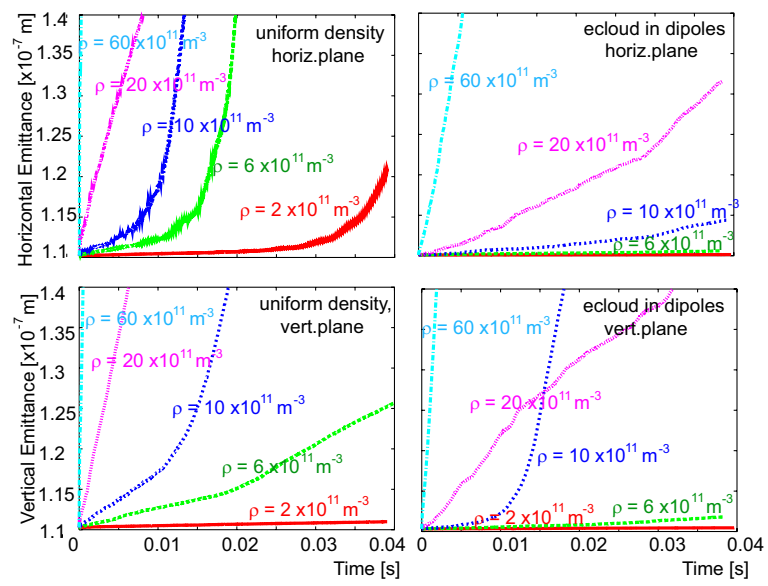

Figure 2: Emittance vs. time for different electron cloud density values, assuming the cloud to be in a field free region or dipoles

\section{BETA FUNCTION VARIATION}

In the original HEADTAIL code, the beam particles are interacting with the electron cloud at a finite number of locations along the ring (" $n$-kick" approximation), where the beta function is assumed to be constant and equal to the average value. Recent modifications allow us to consider different values of $\beta$ at the different interaction points, thereby modeling a variation of the beta function around the ring [7]. Figure 3 shows the effect in the simulation, for two different cloud densities: $\rho_{e}=6 \times 10^{11} \mathrm{~m}^{-3}$ and $3 \times 10^{11} \mathrm{~m}^{-3}$. The first case, with higher electron density, is above the threshold of the TMCI-like instability, which is characterized by a fast blow up of the emittance within a time scale of a synchrotron period and a coherent head-tail motion of the bunch. If only 3 kicks per turn are applied, the beta-function variation affects the results. However, as discussed in previous papers $[8,9]$, we need more than 6 kicks per turn to perform accurate simulations with this set of parameters. Using 10 kicks/turn, changing the $\beta$ pattern has little effect on the result, namely the variable beta function introduces an additional frequency spread, which appears to smoothen the emittance evolution, without a large difference in the instability growth rate.

In the case of a low electron density $\left(3 \times 10^{11} \mathrm{~m}^{-3}\right.$, right picture), i.e., below the threshold of the fast instability, the emittance increases roughly linearly in time. This emittance growth appears to be an incoherent effect, e.g., one due to the excitation of linear and nonlinear resonances $[3,10]$. Here the growth rate is highly affected by the number of kicks per turn and also by the $\beta$ function pattern. The latter can lead to the excitation of additional sets of resonances. For this reason, the $\beta$ function should be sampled for a realistic model of the ring lattice with correct phase advances, in order to represent the nonlinear dynamics accurately. Otherwise, if the betatron phase is sampled in an artificial way, the simulated nonlinear effect of the electron cloud may be far from the real case. 

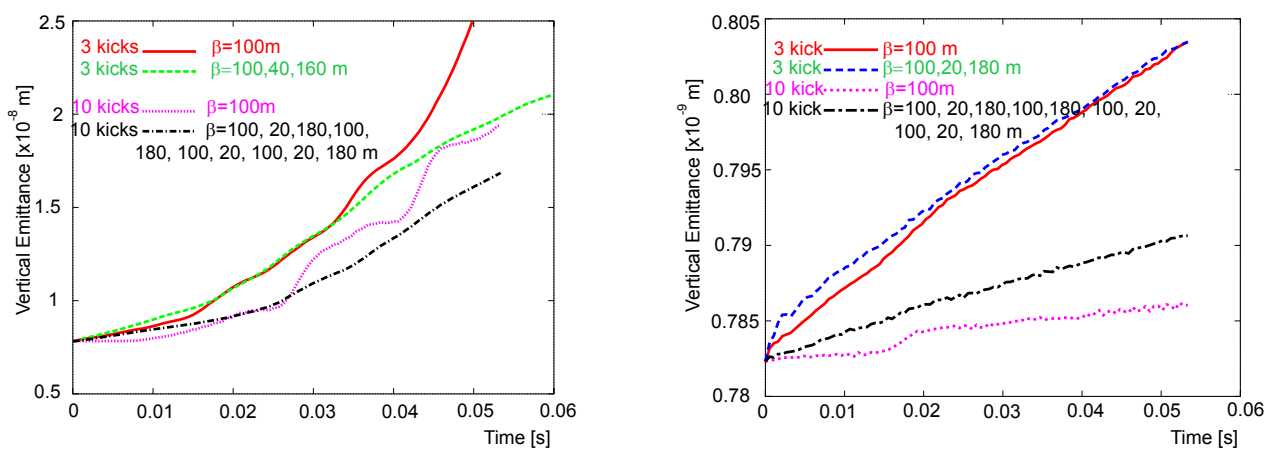

Figure 3: Effect of considering different beta values. Vertical emittance as a function of time, with parameters of LHC at injection and chromaticity $Q=2$, for $\rho_{e}=6 \times 10^{11} \mathrm{~m}^{-3}$ (left) and $\rho_{e}=3 \times 10^{11} \mathrm{~m}^{-3}$ (right).

\section{TOWARDS A REAL LATTICE}

In order to take into account the real $\beta$ variation along the ring including proper phase advances, we implemented in HEADTAIL the FODO cell structure for the CERN SPS. The SPS lattice consists of 108 FODO cells, $64 \mathrm{~m}$ long, and the lattice is represented as a sequence of focusing and defocusing thin-lens quadrupoles, separated by drift spaces or dipoles. The focusing strength is set to yield a phase advance per cell of 90 degrees. The corresponding maximum and minimum beta functions then are about $109 \mathrm{~m}$ and 19 $\mathrm{m}$, respectively, close to the actual values. An extra rotation is applied every $1 / 6$ th of a turn, in order to get the real fractional part of the tune, and to reflect the 6-fold symmetry of the SPS optics. RF focusing is applied once per turn. The chromaticity is set to zero. Eight electron kicks are applied per FODO cell. The lattice elements traversed between kicks are described by standard transport matrices. In view of the computing time required, we run HEADTAIL in the weak-strong approximation, discussed in [3]. The potential created by the electrons is computed only in the first FODO cell, for the eight different locations, then stored and re-used for the following kicks. In this way only incoherent effects can be investigated. A preliminary result for 250 turns indicates a slow emittance growth (Fig. 4), which depends on the electron cloud density. Figure ?? presents a comparison with the results of a constant focusing approximation with different numbers of kicks per turn, for an electron cloud density below the TMCI threshold $\left(\rho_{e}=2 \times 10^{11} \mathrm{~m}^{-3}\right)$.

\section{CONCLUSION AND FUTURE WORK}

Accurate simulations of instability thresholds and slow emittance growth require realistic models of the electron distribution and of the lattice, with several beam-electron interactions per cell. Our weak-strong model can explore the long-term emittance growth below the TMCI threshold. Aspects of a real accelerator lattice are also being implemented in the strong-strong code QUICKPIC, preliminary results of which indicate a significant effect of the dispersion function [11]. Our ultimate objective is a reliable prediction of the long-term emittance growth in the LHC.

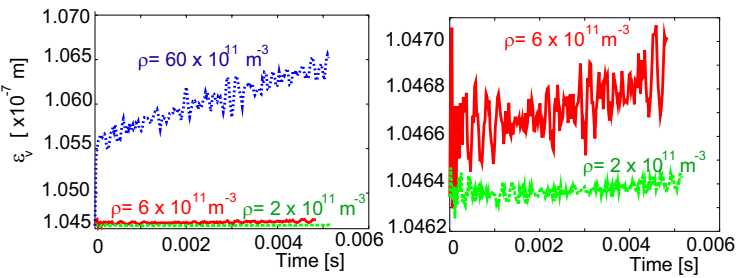

Figure 4: Emittance vs. time by HEADTAIL in weakstrong approximation, with real SPS FODO structure, for different electron densities (left); a zoomed view (right).

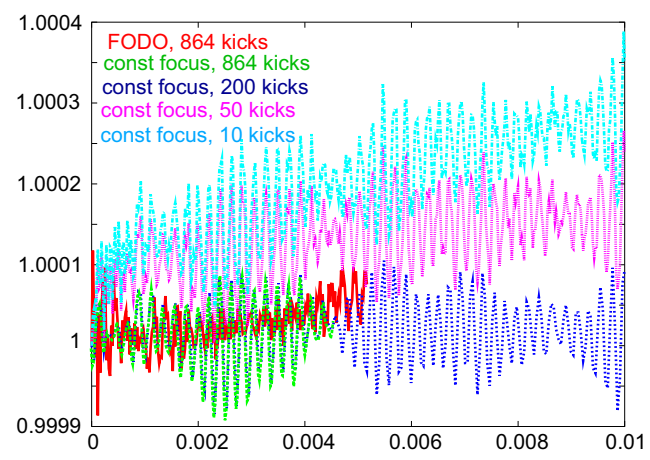

Figure 5: Comparison of SPS emittance growth simulated in weak-strong approximation for the FODO-cell model and for a uniform focusing model with different numbers of kicks, at an electron density of $2 \times 10^{11} \mathrm{~m}^{3}$.

\section{REFERENCES}

[1] J.M. Jimenez et al., LHC Project Report 632 (2003).

[2] G. Rumolo, F. Zimmermann, CERN-SL-Note-2002-036 (2002); see also same authors, PRST-AB 5:121002 (2002).

[3] E. Benedetto et al., "Emittance growth...",these proceedings

[4] K. Ohmi, F. Zimmermann, Phys. Rev. Letters 85, 3831 (2000)

[5] K. Ohmi, F. Zimmermann, E. Perevedentsev, Phys. Rev. E 65, 016502 (2002).

[6] V. Decyk et al., Proc. PAC 2001, Chicago, p. 4005 (2001).

[7] E. Benedetto et al., Proc. ECLOUD’04, Napa (2004).

[8] E. Benedetto et al., Proc. EPAC'04, Lucerne, p. 1831 (2004).

[9] E. Benedetto et al., Proc. ECLOUD’04 Napa (2004)

[10] K. Ohmi, KEK Acc.-Physics Seminar March 2005.

[11] B. Feng, et al. these proceedings. 